Nuclear Physics B353 (1991) 493-518

North-Holland

\title{
THE CANONICAL STRUCTURE OF MAXIMALLY EXTENDED SUPERGRAVITY IN THREE DIMENSIONS
}

\author{
H. NICOLAI \\ II. Institute for Theoretical Physics, Unic ersity of Hamburg, Luruper Chaussee 149, \\ 2000 Hamburg 50, Germany
}

Received 29 October 1990

\begin{abstract}
The algebra of local and rigid symmetries of $N=16$ supergravity in three dimensions is discussed in detail. The conserved charges associated with the rigid $E_{x}$ symmetry are constructed and shown to be physical observables in that they weakly commute with all constraints. The phase-space variables, which render the constraints polynomial. are identified: they are related to the variables used in the $S O(1,2) \times S O(16)$-invariant reformulation of $d=11$ supergravity. This indicates that Ashtekar's approach to canonical gravity can be generalized to higher dimensions if suitable matter degrees of freedom are added to gravity.
\end{abstract}

\section{Introduction}

This paper serves a twofold purpose. First of all, it explains in detail the results of a recent investigation of the algebra of local and rigid symmetries of threedimensional extended supergravities [1]. Secondly, it reveals a possibly important analogy between the "new variables" introduced by Ashtekar in his reformulation of canonical gravity [2] and certain geometrical quantities introduced in refs. [3,4] for the construction of new versions of $d=11$ supergravity [5] with enlarged tangent space symmetry ${ }^{\star}$. This raises the prospect that the approach of ref. [2] can be generalized to higher dimensions, provided suitable matter fields are added to gravity.

In contrast to the pure gravity and supergravity theories in three dimensions, which have received much attention in the past few y'ars [6-9], extended supergravity theories possess not only topological but also propagating degrees of freedom. It is therefore more difficult to solve the constraint equations and to construct observables (in the sense of Dirac) for both the classical and the quantum theories. The Wilson loops with or without insertions of dreibein fields,

* Although this construction has so far only been carried out for $d=11$ supergravity, there is little doubt that other theories of this type admit similar reformulations. 
which constitute the basic observables for pure gravity in three dimensions $[7,8]$, cease to be observables in the presence of matter fields, because the latter act as sources for gravity, and the gravitational curvature thus no longer vanishes on the physical subspace of phase space. As there seems to be no simple modification of the Wilson loop which remedies this defect (this would amount to constructing a curvature-free connection out of the gravitational connection and the matter fields), it is worthwhile to look for other candidates.

One of the main motivations to study extended supergravities in this context derives from the presence of extra noncompact rigid symmetries in these theories [10]. The occurrence of such rigid symmetries is actually not limited to supersymmetric theories, but a generic phenomenon in Kaluza-Klein theories [11] and due to the fact that the scalar sector "remembers" its geometrical origin from a higher-dimensional gravity or supergravity theory. The simplest example is provided by the dimensional reduction of pure Einstein gravity in four dimensions to three dimensions. After an appropriate duality transformation of the Kaluza-Klein vector field, one obtains an $\operatorname{SL}(2, \mathbb{R}) / \mathrm{SO}(2)$ or $\operatorname{SL}(2, \mathbb{R}) / \mathrm{SO}(1,1)$ nonlinear $\sigma$-model coupled to gravity $[11,12]$. The two degrees of freedom of the coset space are naturally related to the two helicity states of the graviton. In the general case, the coset degrees of freedom of the three-dimensional theory are just the bosonic "transverse" degrees of freedom of some (super)gravity theory in higher dimensions, to which the three-dimensional theory is related by dimensional reduction.

The existence of an extra rigid symmetry implies the existence of a conserved current. The associated conserved charges are then given by the integral of the zeroth component of this current over a spacelike hypersurface. As will be shown below, these charges are physical observables in the sense of Dirac. By this we generally mean any functional of the basic fields which weakly commutes with the constraints and does not vanish on the physical subspace. If further observables beyond the conserved charges exist (none are known at this time), even more observables can be constructed by taking successive brackets with these charges. All observables thus belong to representations of the non-compact symmetry group. The corresponding statement for the quantum theory is that the solutions of the Wheeler-DeWitt equation fall into representations of the rigid symmetry group. The observables found here replace (or complement?) the topological observables (Wilson loops) of pure gravity or $N=1$ supergravity in three dimensions.

There is a long history of attempts to quantize gravity in the canonical framework ([13-15]; more recent developments are reviewed in ref. [16]). Recently, an important advance was made by Ashtekar [2], who identified a set of new variables in the phase space of canonical gravity, in terms of which the constraintsunmanageably nonlinear in the metric formalism - become polynomial. This work has opened new avenues towards a possible "pre-geometric" formulation of quantum gravity. In fact, formal solutions of the quantum constraints have been 
found [17]. To be sure, there still remain several important unsolved problems in this approach; apart from the difficulties in interpreting the solutions of ref. [17], the construction of a positive-definite scalar product on the physical Hilbert space of wave-functionals, which is a crucial ingredient in any quantum theory, has not been accomplished so far for the four-dimensional theory. (This problem is relevant to the question of whether the representations of the rigid symmetry to which the observables belong are unitary and hence infinite-dimensional.) On the other hand, a suitable scalar product can be constructed for three-dimensional pure gravity [8]. This is one of the reasons why the study of three-dimensional gravity in this formalism has proved to be a useful exercise. This theory is not only easier to investigate, but in some respects provides a more natural setting for this formulation; for instance, there is no need for a reality constraint unlike in four dimensions (for a clear discussion of this point, see ref. [9]). The program has also been applied to simple supergravity in four dimensions [18] and to matter-coupled theories with Klein-Gordon, Dirac and Yang-Mills fields [19].

In this paper, I will restrict myself to the maximally extended $N=16$ theory in three dimensions [20], first because it is the most symmetric, and secondly, because the corresponding results for other theories are completely analogous. Maximal symmetry is expected to play an important role in the elimination or softening of singularities in the quantum algebra of consiraints. Moreover, the nonlinear structure of the scalar sector typical for theories originating from higher dimensions has not been considered in either [19], nor in the more conventional metric formulation of canonical supergravity [21]. Sect. 2 of this paper contains a brief summary of $N=16$ supergravity. In sects. 3 and 4 , a canonical formulation of this theory will be given. In particular, a set of canonical variables will be identified which renders all constraints as well as the charges associated with the rigid symmetries polynomial, thereby extending the results of ref. [19]^. As will be explained in sect. 5 , the phase-space variables needed here are intimately related to certain geometrical quantities that appear in the $S O(1,2) \times S O(16)$-invariant formulation of $d=11$ supergravity [4] (this construction was based on earlier work [3], where an $\mathrm{SO}(1,3) \times \mathrm{SU}(8)$-invariant version of $d=11$ supergravity was exhibited). There is considerable evidence, that, at least for extended supergravities, the framework proposed in refs. $[3,4]$ is the natural arena for a generalization of Ashtekar's formulation to higher dimensions. Sect. 6 is devoted to a discussion of some important open problems, in particular concerning the question of how to solve the quantum constraints in this theory. It is emphasized that this may require yet a further reduction to $d<3$ space-time dimensions. The $d=2$ theory will be dealt with elsewhere.

^ For simple supergravity in four dimensions, such a set was already found in ref. [18]: however. the elegance of the approach was somewhat compromised by the nonpolynomiality of the reality condition there. 
The conventions and notation used in this paper coincide to a large extent with those of refs. $[1,20]$. The metric has signature $(+--) ; \gamma$-matrices are purely imaginary and obey $\left\{\gamma^{a}, \gamma^{b}\right\}=2 \eta^{a b}$. Curved indices in three dimensions will be denoted by $\mu, \nu, \ldots=0,1,2$ while the letters $i, j, \ldots=1,2$ will be used for spatial indices; flat (Lorentz) indices are denoted by $a, b, \ldots=0,1,2$. To make the distinction completely clear in cases of doubt, I will occasionally put a dot on the index to indicate that it is curved, e.g. in $\gamma^{\dot{0}}=V_{a}^{\dot{0}} \gamma^{a} . \epsilon^{\mu \nu \rho}$ and $\epsilon_{\mu \nu \rho}$ are understood to be tensor densities, whereas the corresponding symbols with flat indices (i.e. $\left.\epsilon^{a b c}\right)$ are always Lorentz tensors; also $\epsilon^{i j} \equiv \epsilon^{\dot{0} i j}$ and $\epsilon_{i j} \equiv \epsilon_{\dot{0} i j}$. In sect. 5, indices $m, n, \ldots=3, \ldots, 10$ will be used to label the internal dimensions of $d=11$ supergravity compactified on $T^{\$}$ down to three dimensions.

In contrast to the conventions used in ref. [1], I will here follow refs. [7-9] and use the dual (and first-order) spin connection

$$
A_{\mu}^{a} \equiv-\frac{1}{2} \epsilon^{a b c} \omega_{\mu b c} .
$$

The gravitationally covariant derivative $\mathscr{Z}_{\mu}$

$$
\mathscr{Z}_{\mu} \epsilon=\left(\partial_{\mu}+\frac{1}{4} \omega_{\mu a b} \gamma^{a b}\right) \epsilon
$$

is consequently replaced by

$$
\mathscr{V}_{\mu} \epsilon=\left(\partial_{\mu}+\frac{1}{2} i \gamma_{a} A_{\mu}^{a}\right) \epsilon
$$

The corresponding field strength reads

$$
F_{\mu v a}=-\frac{1}{2} \epsilon_{a b c} R_{\mu v}^{b c}=\partial_{\mu} A_{\nu a}-\partial_{\nu} A_{\mu a}+\epsilon_{a b c} A_{\mu}^{b} A_{v}^{c}
$$

Observe that $A_{i}^{a}$ and its canonical conjugate constitute the analogue of Ashtekar's variables in three dimensions. The use of $A_{i}^{a}$ as a canonical variable instead of the usual (second-order) spin connection greatly simplifies some of the calculations below. The fact that all three-dimensional supergravities admit this kind of reformulation was already noticed in ref. [22].

\section{2. $N=16$ supergravity and its symmetries}

The $N=16$ theory was constructed in ref. [20]. As already mentioned in sect. 1 , a dual formulation will be used, which is straightforward to derive from the results given in ref. [20]. We will therefore just quote the relevant formulas below, referring the reader to ref. [20] for further details. The physical fields of the three-dimensional $N=16$ theory constitute an irreducible $N=16$ supermultiplet with 128 bosons and 128 fermions transforming as inequivalent fundamental spinor 
representations of $S O(16)$. In addition, the theory contains a dreibein $V_{\mu}^{a}$ and 16 gravitino fields $\psi_{\mu}^{\prime}$, which do not correspond to physical degrees of freedom. All fermion fields are Majorana spinors, i.e. $\bar{x}=\chi^{\mathrm{T}} \mathscr{E}$. The actual construction of the theory is greatly facilitated by exploiting the rigid non-compact $E_{\mathrm{y}}$-invariance of the theory (see the appendix for our $E_{8}$-conventions). The 248 generators of $E_{8}$ are split into 120 generators $X^{I J}=-X^{J l}$ and 128 generators $Y^{A}$ in accordance with the decomposition $248 \rightarrow 120 \oplus 128$ of $E_{8}$ under its $S O(16)$ subgroup, where the indices $I, J, \ldots=1, \ldots, 16$ and $A, B, \ldots=1, \ldots, 128$ (or $\dot{A}, \dot{B}, \ldots=1, \ldots, 128$ ) label the vector representation and the fundamental spinor (or conjugate spinor) representation of SO(16), respectively. The rigid $\mathrm{E}_{\boldsymbol{\gamma}}$-invariance of the theory can be linearly realized in the usual manner by introducing a local SO(16)-invariance. Consequently, the scalars $\mathscr{V}(x)$ are properly described as elements of the coset space $\mathrm{E}_{8(+8)} / \mathrm{SO}(16)$, and the "composite" $\mathrm{SO}(16)$ gauge field $Q_{\mu}^{\prime J}(x)$ is obtained from the $E_{8}$ Lie algebra decomposition

$$
\mathscr{T}^{-1} \partial_{\mu} \mathscr{Z}^{C}=\frac{1}{2} Q_{\mu}^{I J} X^{I J}+P_{\mu}^{A} Y^{A}
$$

The lagrangian is given by

$$
\begin{aligned}
\mathscr{L}= & \frac{1}{4} \epsilon^{\mu \nu \rho} V_{\mu}^{\alpha} F_{\nu \rho a}+\frac{1}{2} \epsilon^{\mu \nu \rho} \bar{\psi}_{\mu}^{I} D_{v} \psi_{\rho}^{I}+\frac{1}{4} V g^{\mu \nu} P_{\mu}^{A} P_{\nu}^{A}-\frac{1}{2} i V \bar{\chi}^{A} \gamma^{\mu}\left(D_{\mu} X\right)^{A} \\
& -\frac{1}{2} V \bar{\chi}^{A} \gamma^{\mu} \gamma^{\nu} \psi_{\mu}^{I} \Gamma_{A \dot{A}}^{I} P_{\nu}^{A}+\frac{1}{8} V(\bar{\chi} X)^{2}-\frac{1}{96} V\left(\bar{\chi} \gamma^{a} \Gamma^{I J} \chi\right)^{2} \\
& -\frac{1}{8} V\left(\bar{\chi} \gamma_{\sigma} \Gamma^{I J} \chi\left(\bar{\psi}_{\rho}^{I} \gamma^{\rho \lambda \sigma} \psi_{\lambda}^{J}-\bar{\psi}_{\rho}^{I} \gamma^{\sigma} \psi^{J \rho}\right)+\bar{\chi} \chi \bar{\psi}_{\rho}^{I} \gamma^{\lambda} \gamma^{\rho} \psi_{\Lambda}^{I}\right)
\end{aligned}
$$

It is invariant under the local supersymmetry variations

$$
\begin{array}{ll}
\delta V_{\mu}^{a}=i \bar{\epsilon}^{I} \gamma^{a} \psi_{\mu}^{I}, & \delta \psi_{\mu}^{I}=D_{\mu} \epsilon^{I}-\frac{1}{4} i \gamma^{\nu} \epsilon^{J} \bar{\chi} \gamma_{\mu v} \Gamma^{I J} \chi, \\
\delta \chi^{A}=\frac{1}{2} i \gamma^{\mu} \epsilon^{I} \hat{P}_{\mu}^{A} \Gamma_{A \dot{A}}^{I}, & \mathcal{Y}^{-1} \delta \gamma^{\prime}=\bar{\epsilon}^{I} \chi^{A} \Gamma_{A \dot{A}}^{I} Y^{A},
\end{array}
$$

where $\hat{P}_{\mu}^{A}$ denotes the supercovariant extension of $P_{\mu}^{A}$

$$
\hat{P}_{\mu}^{A}=P_{\mu}^{A}-\Gamma_{A A}^{I} \bar{\psi}_{\mu}^{I} \chi^{A}
$$

and the derivative $D_{\mu}$ is $\mathrm{SO}(1,2) \times \mathrm{SO}(16)$ covariant:

$$
\begin{aligned}
D_{\mu} \epsilon^{I} & =\left(\partial_{\mu}+\frac{1}{2} i \gamma_{a} A_{\mu}^{a}\right) \epsilon^{I}+Q_{\mu}^{I J} \epsilon^{J} \\
D_{\mu} \chi^{\dot{A}} & =\left(\partial_{\mu}+\frac{1}{2} i \gamma_{a} A_{\mu}^{a}\right) \chi^{\dot{A}}+\frac{1}{4} \Gamma_{\dot{A} \dot{B}}^{I J} Q_{\mu}^{I J} \chi^{\dot{B}}
\end{aligned}
$$


The invariance of $\mathscr{L}$ under local supersymmetry is most conveniently verified in "1.5 order formalism" [23], where the variation of the field $A_{\mu}^{a}$ can be ignored if its equation of motion is substituted into the variation of $\mathscr{L}$. Lagrangian (2.2) is, of course, also invariant under general coordinate transformations in three dimensions, the local Lorentz group $S O(1,2) \cong S L(2, \mathbb{R})$ and $S O(16)$ gauge transformations.

The equations of motion of the physical fields read

$$
V^{-1} D_{\mu}\left(V\left(P^{\mu A}-\bar{\psi}_{{ }^{\prime}}^{l} \gamma^{\mu} \gamma^{\prime \prime} \chi^{\dot{A}} \Gamma_{A \dot{A}}^{I}\right)\right)=\frac{1}{2} V^{-1} \epsilon^{\mu \nu \rho} \bar{\psi}_{\mu}^{I} \psi_{\nu}^{J} \Gamma_{A B}^{I J} P_{\rho}^{B}+\frac{1}{8} i \bar{\chi} \gamma^{\mu} \Gamma^{I J} \chi \Gamma_{A B}^{I J} P_{\mu}^{B}
$$

for the scalars, and

$$
-i \gamma^{\mu} D_{\mu} \chi^{\dot{A}}=\frac{1}{2} \gamma^{\prime \prime} \gamma^{\mu} \psi_{v}^{I} \hat{P}_{\mu}^{A} \Gamma_{A \dot{A}}^{I}-\frac{1}{2}(\bar{\chi} \chi) \chi^{\dot{A}}+\frac{1}{24}\left(\bar{\chi} \gamma^{\mu} \Gamma^{I J} \chi\right) \Gamma_{\dot{A} \dot{B}}^{I J} \gamma_{\mu} \chi^{\dot{B}}
$$

for the spinor degrees of freedom. The Rarita-Schwinger equation is

$$
\epsilon^{\mu \nu \rho} \widehat{D_{v}, \psi_{\rho}^{I}}=\frac{1}{2} \gamma^{v} \gamma^{\mu} \chi^{\dot{A}} \hat{P}_{v}^{A} \Gamma_{A \dot{A}}^{\prime}
$$

where the supercovariant curl is given by

$$
\epsilon^{\mu \nu \rho} \widehat{D_{v}, \psi_{\rho}^{I}} \equiv \epsilon^{\mu \nu \rho} D_{\nu} \psi_{\rho}^{I}+\frac{1}{4} V \bar{\chi} \gamma^{\nu^{\prime}} \Gamma^{I J} \chi\left(\gamma^{\mu} \psi_{v}^{J}-\delta_{v}^{\mu} \gamma^{\lambda} \psi_{\lambda}^{J}\right)
$$

The dual field $A_{\mu}^{a}$ can be expressed in terms of the other fields by solving its equation of motion

$$
\frac{1}{2} \epsilon^{\mu l^{\prime} \rho} D_{\imath} V_{\rho}^{a}=\frac{1}{4} i \epsilon^{\mu v^{\prime} \rho} \bar{\psi}_{l^{\prime}}^{I} \gamma^{a} \psi_{\rho}^{I}-\frac{1}{4} V V_{a}^{i} \bar{\chi} \chi
$$

It has already been emphasized that the distinguishing feature of three-dimensional (super)gravities, which originate from some higher-dimensional (super)gravity theory, is the presence of extra rigid symmetries and associated conserved currents. For $N=16$ supergravity, this current is [1]

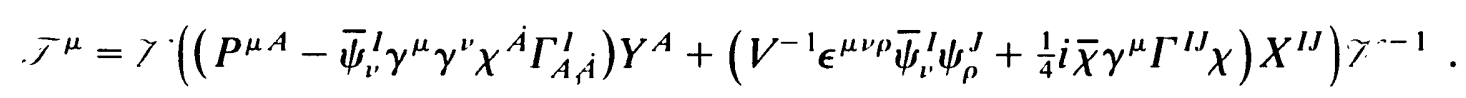

To verify that this current is indeed conserved, i.e. $\partial_{\mu}\left(V \bar{J}^{\mu}\right)=0$, one has to make use of eq. (2.1) and the equations of motion (2.6)-(2.9). Note that the expression for the conserved current contains no quartic or higher-order terms in the fermions. This is to be contrasted with the expressions for the hamiltonian 
constraint and the supersymmetry generators which will be presented below. The absence of higher-order fermionic terms in the $\mathrm{E}_{8}$-current is, of course, in accord with one's expectation that the noncompact symmetry acts linearly on the fermions. The associated $\mathrm{E}_{8}$-charges

$$
\mathscr{Q}=\int V \mathscr{T}^{\dot{0}} \mathrm{~d}^{2} x
$$

are conserved if the fields satisfy their respective equations of motion and vanish at spatial infinity or satisfy periodic boundary conditions. Occasionally, the $\mathrm{E}_{\mathbf{8}}$-indices will be written out explicitly,

$$
\mathscr{Q}_{\alpha \beta}=\frac{1}{2} \mathscr{Q}^{I J} X_{\alpha \beta}^{I J}+\mathscr{Q}^{A} Y_{\alpha \beta}^{A}
$$

where Greek indices $\alpha, \beta, \ldots=1, \ldots, 248$ collectively denote the $\mathrm{E}_{8}$ indices $(A, I J), \ldots$ in the fundamental representation. Below, we will consider the algebra generated by the constraints and $\mathscr{Q}$. However, the (on-shell) invariance of these charges can also be checked directly. Inserting the supersymmetry variations given above and making use of the equations of motion once more, it is not difficult to show that

$$
\delta\left(V \mathscr{T}^{\mu}\right)=\partial_{\nu}\left(V \mathscr{M}^{\nu \mu}\right)
$$

where

$$
\mathscr{M}^{\mu \nu}=\mathscr{V}^{-1}\left(\bar{\epsilon}^{I} \gamma^{\mu \nu} \chi^{\dot{A}} \Gamma_{A \dot{A}}^{I} Y^{A}+2 V^{-1} \epsilon^{\mu \nu \rho} \bar{\psi}_{\rho}^{I} \epsilon^{J} X^{I J}\right) \mathscr{T}^{-1}
$$

Therefore, the $E_{8}$-charges are supersymmetric (i.e. $\left.\delta \subsetneq=0\right)$ under the same conditions that ensure their conservation.

\section{Canonical formalism}

Let us now proceed to the hamiltonian formulation of the theory. The dual formulation presented in the preceding section is far more convenient for this purpose than the more conventional treatment of canonical supergravity in second-order formalism [21]. Especially the brackets involving the gravitational phase-space variables are considerably simplified. It has already been emphasized in sect. 1 that matter-coupled theories present new features. In extended supergravities, the matter sectors are highly nonlinear owing to their geometrical origin and severely constrained by the symmetries. The canonical formulation of the matter sector requires the proper treatment of nonlinear coset space $\sigma$-models coupled to gravity, a topic which has not been extensively discussed in the literature so far. 
At this point, it is appropriate to introduce some further notation which will allow us to make the decoupling of the Lagrange multiplier field $V_{0}^{a}$ from all canonical expressions completely explicit. In contrast to ref. [1], no special gauge will be chosen for the dreibein. This has the advantage that we will be able to realize the full local Lorentz group in phase space [24]. The spatial part of the metric is

$$
h_{i j} \equiv V_{i}^{a} V_{j a}
$$

(Unlike in ref. [1], I do not switch to a positive-definite metric here; this accounts for some sign differences between the formulas here and in ref. [1]). The inverse metric will be denoted by $h^{i j}$ and is related to the inverse of the full space-time metric by

$$
h^{i j}=g^{i j}-\frac{g^{\dot{0} i} g^{\dot{0} j}}{g^{\dot{0} \dot{0}}}
$$

To clarify whether an index has been raised with $h^{i j}$ or $g^{i j}$, I will either write out the contractions explicitly, or else put a hat on the tensor under consideration. For instance,

$$
\hat{V}_{a}^{i} \equiv h^{i j} V_{j a}=V_{a}^{i}-\frac{g^{\dot{0} i} g^{\dot{0} j}}{g^{\dot{0} \dot{0}}} V_{j a}
$$

The determinant $h \equiv \operatorname{det} h_{i j}$ is related to the full metric determinant by

$$
h=V^{2} g^{i \dot{0}} \text {. }
$$

To have a complete basis of vector fields, it is furthermore necessary to define a timelike vector field $n^{a}$ [24], viz.

$$
n_{a} \equiv V_{a}^{i} / \sqrt{g^{\dot{0} \dot{0}}}
$$

which satisfies

$$
n^{a} n_{a}=1, \quad n^{a} V_{i a}=0
$$

Alternatively, $n^{a}$ can be defined through these properties. Thus, despite appearances, $n^{a}$ does not depend on $V_{i}^{a}$ but only on $V_{i}^{a}$. Comparison of eqs. (3.4) and (3.5) immediately leads to

$$
V V_{a}^{i}=\frac{1}{2} \epsilon_{a b c} \epsilon^{i j} V_{i}^{b} V_{j}^{c}=\sqrt{h} n_{a}
$$


which can be solved for $\sqrt{h}$

$$
\sqrt{h}=\frac{1}{2} \epsilon_{a b c} \epsilon^{i j} n^{a} V_{i}^{b} V_{j}^{c}=\frac{1}{2} \epsilon_{a b c} \epsilon^{i j} n^{a} \hat{V}_{i}^{b} \hat{V}_{j}^{c}
$$

Note that neither $\sqrt{h}$ nor the vector $n^{a}$ are polynomial functions of $V_{i}^{a}$; only the combination appearing in eq. (3.7) is. The gauge employed in ref. [1] corresponds to $n^{a}=(1,0,0)$. I will also write $\gamma_{\perp} \equiv n_{a} \gamma^{a}$.

It is a well-known feature of gauge theories that their invariance under local symmetries is reflected in the existence of first-class constraints [13]. These are also the canonical generators of infinitesimal gauge transformations in phase space. More explicitiy, one obtains a constraint for every field whose time derivative is absent from $\mathscr{L}$ and whose conjugate momentum thus vanishes. In the lagrangian, these fields appear as Lagrange multipliers. In the case at hand, they are $V_{\dot{0}}^{a}, A_{\dot{0}}^{a}$, $\psi_{0}^{I}$ and $Q_{0}^{I I}$, which are associated with local translations, local Lorentz rotations, local supersymmetry and local $\mathrm{SO}(16)$-transformations, respectively. Consistency then requires that all of these field components drop out of the expressions for the canonical generators, since otherwise the conjugate momenta could not be consistently put equal to zero.

Varying $\mathscr{L}$ with respect to $V_{0}^{a}$ yields the "Poincaré generators"

$$
\mathscr{H}_{a} \equiv \delta \mathscr{L} / \delta V_{\dot{0}}^{a}
$$

The generators of infinitesimal coordinate transformations are obtained from these after contraction with the dreibein

$$
\mathscr{H}_{\mu} \equiv V_{\mu}^{a} \mathscr{H}_{a} .
$$

The explicit expressions for them are somewhat complicated (although straightforward to derive from eq. (1.2)) and will be given only after the identification of the proper phase-space variables. In order to eliminate the dependence on the Lagrange multiplier $V_{i}^{a}$, a slightly different decomposition of $\mathscr{H}_{a}$ must be employed [15,24], viz.

$$
\mathscr{H}_{a}=n_{a} \mathscr{H}_{\perp}+\hat{V}_{a}^{i} \mathscr{H}_{i}, \quad \mathscr{H}_{\perp}=\frac{1}{\sqrt{g^{\dot{0} \hat{0}}}} g^{\dot{0} \mu} \mathscr{H}_{\mu} .
$$

It is a well-known feature of canonical gravity that timelike and spacelike constraints play a different role. For $\mu=i$, we get the generators of spatial diffeomorphisms. Requiring them to vanish is analogous to Gauss' law in the canonical formulation of gauge theories and simply restricts the space of configurations. These are also the constraints which are "easy" to solve. The timelike constraint, on the other hand, generates time translations and therefore contains dynamical 
information; its quantum version, the Wheeler-DeWitt equation, is the "Schrödinger equation" of quantum gravity.

The generator of local supersymmetry transformations can be read off directly from eq. (2.8)

$$
\mathscr{F}^{I} \equiv-\frac{\delta \mathscr{Z}}{\delta \bar{\psi}_{0}^{I}}=-\epsilon^{i j} \widehat{D_{i} \psi_{j}^{I}}+\frac{1}{2} V \gamma^{\mu} \gamma^{\dot{0}} \chi^{\dot{A}} \Gamma_{A \dot{A}}^{I} \widehat{P_{\mu}^{A}}
$$

Again, its expression in terms of the proper phase-space variables is deferred until sect. 4. The generator of the Lorentz transformations reads

$$
L_{a} \equiv \frac{\delta \mathscr{Z}}{\delta A_{\dot{0}}^{a}}=\frac{1}{2} \epsilon^{i j} D_{i} V_{j}^{a}-\frac{1}{4} i \epsilon^{i j} \bar{\psi}_{i}^{I} \gamma_{a} \psi_{j}^{I}+\frac{1}{4} V V_{a}^{\dot{0}} \bar{\chi} \chi
$$

There is a further constraint associated with the invariance of $\mathscr{L}$ under local SO(16)-transformations. We could derive it simply by varying $\mathscr{L}$ with respect to $Q_{0}^{I J}$ in a first-order formulation, where the gauge fields $Q_{\mu}^{I J}$ are treated as independent fields, whose expression in terms of the physical fields is determined from their equations of motion. Although this would be the analogue of the first-order formulation which we have employed for the gravitational sector, we prefer to follow a different path here by invoking the second-order formalism for the scalar sector, see below. In accordance with the general theory, we interpret the vanishing of the constraints as weak equalities [13]

$$
\mathscr{H}_{\mu}(x) \approx 0, \quad \mathscr{f}^{\prime}(x) \approx 0, \quad L_{a}(x) \approx 0, \quad \phi^{I J}(x) \approx 0 .
$$

In the quantum theory, the constraints become opcrator constraints on the physical states.

Before evaluating the canonical algebra of the constraints, we must now identify the proper phase-space variables and compute their canonical brackets. Due to the presence of second-class constraints, some of the basic Poisson brackets must be modified [13]. Let us first consider the fermionic brackets. These are given by

$$
\begin{gathered}
\left\{\psi_{i}^{\prime}(x), \bar{\psi}_{j}^{J}(y)\right\}=\epsilon_{i j} \delta^{I J} \delta^{(2)}(x, y), \\
\left\{\chi^{\dot{A}}(x), \bar{\chi}^{\dot{B}}(y)\right\}=i\left(V g^{\dot{0} \dot{0}}\right)^{-1} \gamma^{\dot{0}} \delta^{\dot{A} \dot{B}} \delta^{(2)}(x, y),
\end{gathered}
$$

where spinor indices have been suppressed and the variables $x, y$ parametrize the spacelike hypersurface. Eq. (3.16) can be alternatively written in terms of the 
variables defined before

$$
\left\{\chi^{\dot{A}}(x), \bar{\chi}^{\dot{B}}(y)\right\}=\frac{i}{\sqrt{h}} \gamma_{\perp} \delta^{\dot{A} \dot{B}} \delta^{(2)}(x, y),
$$

which reduces to the corresponding result of ref. [1] with the choice $n^{a}=(1,0,0)$.

To write out the brackets for the gravitational fields, define

$$
\Pi_{a}^{i} \equiv \frac{\delta \mathscr{L}}{\delta \partial_{\dot{0}} A_{i}^{a}}=\frac{1}{2} \epsilon^{i j} V_{j a}
$$

This variable is directly related to the variables discussed at the beginning of this section. For example,

$$
\sqrt{h} \hat{V}_{a}^{i}=-2 \epsilon_{a b c} n^{b} \Pi^{c i}
$$

from which it immediately follows that $4 \Pi^{a i} \Pi_{a}^{j}=h h^{i j}$, so $\Pi_{a}^{i}$ can be thought of as the square root of the densitized inverse of the spatial metric (3.1). The canonical brackets are now given by

$$
\left\{A_{i}^{a}(x), \Pi_{b}^{j}(y)\right\}=\delta_{b}^{a} \delta_{i}^{j} \delta^{(2)}(x, y)
$$

and, of course, $\left\{\Pi_{a}^{i}, \Pi_{b}^{j}\right\}=0$. The field $A_{i}^{a}$ also has nonvanishing brackets with the timelike vector $n^{a}$ introduced above, which can be determined from eqs. (3.7) and (3.20). Although this relation will not be needed in the remainder, it is given here for completeness:

$$
\left\{A_{i}^{a}(x), n^{b}(y)\right\}=\frac{2}{\sqrt{h}}\left(\delta_{c}^{b}-n^{b} n_{c}\right) \epsilon^{a c d} V_{i d} \delta^{(2)}(x, y) .
$$

This is consistent with the orthogonality condition in eq. (3.6). Furthermore,

$$
\begin{aligned}
\left\{A_{i}^{a}(x), A_{j}^{b}(y)\right\} & =-\left(g^{\dot{0} \dot{0}}\right)^{-1} \epsilon_{i j} V^{a \dot{0}} V^{b \dot{0}} \bar{\chi} \chi \delta^{(2)}(x, y) \\
& =-\epsilon_{i j} n^{a} n^{b} \bar{\chi} \chi \delta^{(2)}(x, y), \\
\left\{A_{i}^{a}(x), \chi^{\dot{A}}(y)\right\} & =-\epsilon^{a b c} V_{i c}\left(V g^{\dot{0} \dot{0}}\right)^{-1} \gamma^{\dot{0}} \gamma_{b} \chi^{\dot{A}} \delta^{(2)}(x, y) \\
& =\frac{1}{\sqrt{h}} \epsilon^{a b c} V_{b i} \gamma_{\perp} \gamma_{c} \chi^{\dot{A}} \delta^{(2)}(x, y),
\end{aligned}
$$

whereas $\left\{A_{i}^{a}, \psi_{j}^{L}\right\}=0$. The nonvanishing bracket between $A_{i}^{a}$ and $\chi^{\dot{A}}$ above 
ensures for instance that

$$
\left\{A_{u}^{a d}(x), V \bar{\chi} \gamma^{i j} \Gamma^{\prime \prime} \chi(y)\right\}=0,
$$

a result which will be useful below. In ref. [1] the brackets involving the spinors $\chi^{A}$ were found to simplify somewhat after the redefinition $\chi^{A} \rightarrow h^{1 / 4} \chi^{A}$. Unfortunately, absorbing the factor $h^{1 / 4}$ into $\chi^{-4}$ here does not eliminate the $x$-dependence on the right-hand side of eq. (3.17) because $\gamma_{1}$ still depends on $x$ through $n^{\prime 2}$ (this dependence disappears only in the gauge $n^{a}=(1,0,0)$ ). Moreover, the brackets with $A_{3}^{i z}$ cannot be made to vanish in this fashion; rather,

$$
\left\{A_{i}^{a}(x), h^{1 / 4} \chi^{-i}(y)\right\}=\frac{i}{\sqrt{h}} n^{a} \gamma_{i}\left(h^{1 / 4} \chi^{i}\right) \delta^{(2)}(x, y) .
$$

Because the right-hand side vanishes when contracted with $V_{j a}$, there is no inconsistency with the corresponding result of ref. [1].

The scalar sector is somewhat more complicated, and requires some further notation. To derive the canonically conjugate momenta, we temporarily parameterize $\gamma^{\prime}$ in terms of the 128 physical scalars $\varphi^{A}$ and 120 additional fields $\varphi^{\prime \prime}$, which are gauge degrees of freedom, i.e.

$$
\gamma^{\prime}(x)=\exp \left(\varphi^{A}(x) Y^{A}+\frac{1}{2} \varphi^{I J}(x) X^{I J}\right)
$$

This parametrization will no longer be needed in the analysis of the canonical constraint algebra below. It is furthermore useful to define $E_{\mathrm{x}}$ matrices $M=$ $M_{\alpha \beta}\left(\varphi^{-A} \cdot \varphi^{J}\right)$ by

$$
\begin{aligned}
& y^{-1} \frac{\partial y^{\cdot}}{\partial \varphi^{A}}=\left(M^{-1}\right)^{A B} Y^{B}+\frac{1}{2}\left(M^{-1}\right)^{A \cdot K L} X^{K L}, \\
& y^{-1} \frac{\partial \zeta^{\prime}}{\partial \varphi^{I J}}=\left(M^{-1}\right)^{I J \cdot B} Y^{B}+\frac{1}{2}\left(M^{-1}\right)^{I J \cdot K L} X^{K L} .
\end{aligned}
$$

The inierse matrices are then defined by

$$
\begin{gathered}
M^{A C}\left(M^{-1}\right)^{C B}+\frac{1}{2} M^{A, K L}\left(M^{-1}\right)^{K L, B}=\delta^{A B}, \\
M^{I J, C}\left(M^{-1}\right)^{C B}+\frac{1}{2} M^{I J, K L}\left(M^{-1}\right)^{K L, B}=0, \\
M^{I J, C}\left(M^{-1}\right)^{C, K L}+\frac{1}{2} M^{I J, P Q}\left(M^{-1}\right)^{P Q, K L}=\delta_{K L}^{I J} .
\end{gathered}
$$


A useful fact is

$$
\frac{\partial}{\partial \varphi^{\alpha}}\left(y^{-1} \frac{\partial Z^{\circ}}{\partial \varphi^{\beta}}\right)-(\alpha \leftrightarrow \beta)=f^{\alpha \beta \gamma}\left(y^{-1} \frac{\partial \gamma^{\nu}}{\partial \varphi^{\gamma}}\right)
$$

where the notation $\alpha, \beta, \gamma, \ldots$ for the $\mathrm{E}_{\boldsymbol{\gamma}}$ indices $A,[\boldsymbol{J}], \ldots$ has already been introduced before, and $f^{\alpha \beta \gamma}$ denote the $\mathrm{E}_{8}$ structure constants. The momenta canonically conjugate to $\varphi^{A}$ and $\varphi^{\prime J}$ will be denoted by $\Pi^{A}$ and $\Pi^{I J}$, respectively. Using the above definitions, we obtain

$$
\begin{aligned}
\Pi^{A}=\frac{\delta \mathscr{L}^{\prime}}{\delta \partial_{\dot{0}} \varphi^{A}}= & \frac{1}{2} V\left(M^{-1}\right)^{A B}\left(P^{\dot{0} B}-\bar{\psi}_{\mu}^{K} \gamma^{\dot{0}} \gamma^{\mu} \chi^{\dot{B}} \Gamma_{B \dot{B}}^{K}\right) \\
& +\frac{1}{2}\left(M^{-1}\right)^{A, K L}\left(-\frac{1}{4} i V \bar{\chi} \gamma^{\dot{\theta}} \Gamma^{K L} \chi-\epsilon^{i j} \bar{\psi}_{i}^{K} \psi_{j}^{L}\right) \\
\Pi^{I J}=\frac{\delta \mathscr{Z}}{\delta \partial_{\dot{0}} \varphi^{I J}}= & \frac{1}{2}\left(M^{-1}\right)^{I J \cdot B}\left(P^{\dot{0} B}-\bar{\psi}_{\mu}^{K} \gamma^{\dot{0}} \gamma^{\mu} \chi^{\dot{B}} \Gamma_{B \dot{B}}^{K}\right) \\
& +\frac{1}{2}\left(M^{-1}\right)^{I J \cdot K L}\left(-\frac{1}{4} i V \bar{\chi} \gamma^{\dot{0}} \Gamma^{K L} \chi-\epsilon^{i j} \bar{\psi}_{i}^{K} \psi_{j}^{L}\right) .
\end{aligned}
$$

Making use of eq. (3.28), we can invert these relations to get

$$
\tilde{P^{A}} \equiv V\left(P^{\dot{0} A}-\bar{\psi}_{\mu}^{\prime} \gamma^{\dot{0}} \gamma^{\mu} \chi^{\dot{A}} \Gamma_{A \dot{A}}^{\prime}\right)=2 M^{A B} \Pi^{B}+M^{A, K L} \Pi^{K L}
$$

These phase-space variables are much more convenient than the canonical momenta $\Pi^{A}$ because their Poisson brackets turn out to be rather simple. In the following section, it will be shown that the $\tilde{P}^{A}$ and the variables discussed below are entirely sufficient to express all canonical quantities of interest.

Furthermore, we define

$$
\phi^{I J} \equiv 2 M^{I J, B} \Pi^{B}+M^{I J, K L} \Pi^{K L}+\frac{1}{4} i V \bar{\chi} \gamma^{\dot{0}} \Gamma^{I J} \chi+\epsilon^{i j} \bar{\psi}_{i}^{I} \psi_{j}^{J}
$$

Inspection shows that $\phi^{I J}=0$ on account of eqs. (3.28) and (3.30). In accordance with the remarks above, the vanishing of $\phi^{I J}$ should be interpreted as a weak equality. This constraint is, of course, just a consequence of the invariance of the theory under local SO(16)-transformations, which allows us to gauge away the scalar fields $\varphi^{\prime J}$. The constraints $\phi^{I J}$ are therefore the canonical generators of SO(16) gauge transformations.

From the basic Poisson bracket

$$
\left\{\varphi^{A}(x), \Pi^{B}(y)\right\}=\delta^{A B} \delta^{(2)}(x, y)
$$


and the relations given above one derives

$$
\begin{gathered}
\left\{\tilde{P}^{A}(x), Y^{-}(y)\right\}=-2\left(Y^{A} Y^{A}\right) \delta^{(2)}(x, y) \\
\left\{\tilde{P^{A}}(x), y^{-1}(y)\right\}=+2\left(Y^{A} y^{-1}\right) \delta^{(2)}(x, y) .
\end{gathered}
$$

The advantage of using $\tilde{P}^{-4}$ as phase-space variables rather than $P^{\dot{0} A}$, is that their brackets with the fermionic fields vanish:

$$
\left\{\tilde{P} A(x), \chi^{\dot{A}}(y)\right\}=\left\{\tilde{P^{A}}(x), \psi_{m}^{I}(y)\right\}=0,
$$

a property which does not hold for $P^{i A}$. From eq. (3.31) for $\tilde{P}^{A}$ and (3.32), one deduces

$$
\left\{\tilde{P}^{A}(x), \tilde{P}^{B}(y)\right\}=\Gamma_{A B}^{I J}\left(-\phi^{I J}+\frac{1}{4} i V \bar{\chi} \gamma^{\dot{0}} \Gamma^{I J} \chi+\epsilon^{i j} \bar{\psi}_{i}^{I} \psi_{j}^{J}\right) \delta^{(2)}(x, y) .
$$

The fermionic bilinears on the right-hand side will be essential for the closure of the constraint and charge algebras later on. The defining relation (2.1) for $P_{i}^{A}$ and $Q_{i}^{I J}$ in conjunction with eqs. (3.34) and (3.35) leads to

$$
\begin{gathered}
\left\{\tilde{P}^{A}(x), P_{i}^{B}(y)\right\}=2 D_{i}^{A B} \delta^{(2)}(x, y)=\left(2 \delta^{A B} \partial_{i}+\frac{1}{2} Q_{i}^{I J} \Gamma_{A B}^{I J}\right) \delta^{(2)}(x, y) \\
\left\{\tilde{P}^{A}(x), Q_{i}^{I J}(y)\right\}=\Gamma_{A B}^{I J} P_{i}^{B} \delta^{(2)}(x, y)
\end{gathered}
$$

The canonical generators $\phi^{I J}$ of $S O(16)$ gauge transformations obey

$$
\left\{\phi^{I J}(x), \phi^{K L}(y)\right\}=-4 \delta^{[L[I} \phi^{J] K]}(x) \delta^{(2)}(x, y)
$$

From the above brackets, it is straightforward to verify that they do generate SO(16)-transformations; for instance

$$
\begin{gathered}
\left\{\phi^{I J}(x), Y^{\prime}(y)\right\}=-\left(\mathcal{Y} X^{I J}\right) \delta^{(2)}(x, y), \\
\left\{\phi^{I J}(x), \chi^{\dot{A}}(y)\right\}=\frac{1}{2} \Gamma_{\dot{A} \dot{B}}^{I J} \chi^{\dot{B}} \delta^{(2)}(x, y) .
\end{gathered}
$$

To summarize, the quantities whose brackets have been listed above will be our basic phase-space variables from now on. This also means that the explicit parametrization (3.26) used in their derivation can now be discarded.

\section{The algebra of constraints and charges}

Having identified the proper phase-space variables, one can now express the constraints in terms of them and, by means of the canonical brackets computed 
above, determine their algebra. After some rearrangement, the generators of space-dependent supersymmetry transformations become

$$
\mathscr{S}^{I}=\epsilon^{i j} D_{i} \psi_{j}^{I}-\frac{1}{2} \chi^{\dot{A}} \Gamma_{A A}^{I} \tilde{P}^{A}-i \Pi_{a}^{i} \gamma^{a} \chi^{\dot{A}} \Gamma_{A \dot{A}}^{I} P_{i}^{A}+\frac{1}{2} \epsilon_{a b c} \gamma^{a} \psi_{i}^{J} \Pi^{i b} \bar{\chi} \gamma^{c} \Gamma^{I J} \chi
$$

The generators of Lorentz-transformations now read

$$
L_{a}=D_{i} \Pi_{a}^{i}-\frac{1}{4} i \epsilon^{i j} \bar{\psi}_{i}^{\prime} \gamma_{a} \psi_{j}^{\prime}+\frac{1}{4} V V_{a}^{\dot{0}} \bar{\chi} \chi
$$

The derivation of the generators $\mathscr{H}_{\mu}$ is somewhat more cumbersome. After some calculation, one finds

$$
\mathscr{H}_{i}=-\Pi^{a j} F_{i j a}+\epsilon^{j k} \bar{\psi}_{i}^{I} D_{j} \psi_{k}^{I}-\frac{1}{2} \tilde{P}^{A} P_{i}^{A}+\frac{1}{2} i V \bar{\chi}^{\dot{A}} \gamma^{\dot{0}} D_{i} \chi^{A}-\bar{\psi}_{i}^{I} \cdot \mathscr{P}^{I}
$$

Observe that the $\bar{\psi} D \psi$-term actually cancels in this expression ${ }^{\star}$. From this term it is also evident that $\mathscr{H}_{i}$ does not just generate spatial diffeomorphisms. The explicit calculation reveals that, in addition to a local supersymmetry transformation with parameter $\xi^{i} \psi_{i}^{l}, \xi^{i} \mathscr{H}_{i}$ generates local $\mathrm{SO}(16)$ and local Lorentz transformations with parameters $\frac{1}{2} \xi^{i} Q_{i}^{I J}$ and $\xi^{i} A_{i}^{a}$, respectively. It is therefore sometimes convenient to define another generator $\mathscr{H}_{i}^{\prime}$ by subtracting the other constraints present in (4.3). Explicitly,

$$
\mathscr{H}_{i}^{\prime} \equiv \mathscr{H}_{i}+\bar{\psi}_{i}^{I} \mathscr{H}^{I}-\frac{1}{2} Q_{i}^{I J} \phi^{I J}+A_{i}^{a} L_{a} .
$$

It is evident that these constraints are polynomial functions of the canonical variables introduced in the foregoing section. Although the nondegeneracy of the metric was implicitly used at intermediate steps in the calculation, one can at this point abandon this requirement and let $\Pi_{a}^{i}$ vary freely, in complete analogy with the conclusions of $[7-9,19]$.

To derive the explicit form of the Hamiltonian constraint is more tedious. After some algebra, one obtains

$$
\begin{aligned}
\mathscr{H}_{\dot{0}}= & \frac{1}{4} \epsilon^{a b c} V V_{a}^{i} V_{b}^{j} F_{i j c}-\frac{1}{2} i V \bar{\chi}^{\dot{A}} \gamma^{i} D_{i} \chi^{\dot{A}}-\frac{1}{4}\left(V g^{\dot{0} \dot{0}}\right)^{-1}\left(\tilde{P}^{A}-V g^{i \dot{0}} P_{i}^{A}\right)^{2} \\
& +\frac{1}{4} V g^{i j} P_{i}^{A} P_{j}^{A}-\frac{1}{2} V P_{i}^{A} \bar{\chi}^{\dot{A}} \gamma^{j} \gamma^{i} \psi_{j}^{I} \Gamma_{A \dot{A}}^{I}-\frac{1}{2}\left(g^{\dot{0} \dot{0}}\right)^{-1}\left(\tilde{P}^{A}-V g^{\dot{0} i} P_{i}^{A}\right) \bar{\chi}^{\dot{A}} \gamma^{j} \gamma^{\dot{0}} \psi_{j}^{I} \Gamma_{A \dot{A}}^{I} \\
& -\frac{1}{4} i\left(g^{\dot{0} \hat{0}}\right)^{-1} \bar{\chi} \chi \epsilon^{i j} \bar{\psi}_{i}^{I} \gamma^{\dot{0}} \psi_{j}^{I}+\frac{1}{4} V \bar{\chi} \gamma^{i} \Gamma^{I J} \chi \bar{\psi}_{i}^{I} \gamma^{j} \psi_{j}^{J}-\frac{1}{2} V\left(g^{\dot{0} \dot{0}}\right)^{-1} g^{\dot{0} i \bar{\chi}} \gamma^{j} \Gamma^{I J} \chi \bar{\psi}_{i}^{I} \gamma^{\dot{0}} \psi_{j}^{J} \\
& +\frac{1}{4} V\left(g^{\dot{0} \dot{0}}\right)^{-1} g^{i j} \bar{\chi} \gamma^{\dot{0}} \Gamma^{I J} \chi \bar{\psi}_{i}^{I} \gamma^{\dot{0}} \psi_{j}^{J}+\frac{1}{8} V(\bar{\chi} \chi)^{2}-\frac{1}{96} V\left(\bar{\chi} \gamma^{a} \Gamma^{I J} \chi\right)^{2}
\end{aligned}
$$

Notice that the variables $A_{0}^{a}, \psi_{\dot{0}}^{l}$ and $Q_{\dot{0}}^{I J}$ do drop out of these expressions, as is

\footnotetext{
« The corresponding term has not been correctly given in eq. (26) of ref. [1].
} 
required by consistency. Use of the canonical variables $\tilde{P}^{A}$ is essential here, because they completely absorb the unwanted gravitino components $\psi_{\dot{0}}^{I}$. On the other hand, the Lagrange multiplier field $v_{i}^{a}$ is not yet decoupled in the above form. In order to eliminate it, we must use eq. (3.11) in the form

$$
\mathscr{H}_{\perp}=\sqrt{g^{\dot{0} \dot{0}}}\left(\mathscr{H}_{\dot{0}}+\frac{g^{\dot{0} i}}{g^{\dot{0} \dot{0}}} \mathscr{H}_{i}\right) .
$$

Some further calculations show that, indeed, the hamiltonian acquires a nicer form in this way.

$$
\begin{aligned}
\ddot{H}_{\perp}= & \frac{1}{4} \epsilon^{a b c} \sqrt{h} \hat{V}_{a}^{i} \hat{V}_{b}^{i} F_{i j c}-\frac{1}{2} i \sqrt{h} \hat{V}_{a}^{i} \bar{\chi}^{\dot{A}} \gamma^{a} D_{j} \chi^{A}-\frac{1}{4}(1 / \sqrt{h}) \tilde{P} A \tilde{P}^{A}+\frac{1}{4} \sqrt{h} h^{i j} P_{i}^{A} P_{j}^{A} \\
& -\frac{1}{2} \hat{V}_{a}^{i} \bar{\chi}^{\dot{A}} \gamma^{a} \gamma_{\perp} \psi_{i}^{I} \tilde{P}^{A} \Gamma_{A A}^{I}-\frac{1}{2} \sqrt{h} \hat{V}_{a}^{i} \hat{V}_{b}^{j} \bar{\psi}_{i}^{I} \gamma^{b} \gamma^{a} \chi^{A} P_{j}^{A} \Gamma_{A A}^{I} \\
& +\frac{1}{4} \sqrt{h} \hat{V}_{a}^{l} \hat{V}_{b}^{j} \bar{\psi}_{i}^{I} \gamma^{b} \psi_{j}^{J} \bar{\chi} \gamma^{a} \Gamma^{I J} \chi+\frac{1}{4} \sqrt{h} h^{i j} \bar{\psi}_{i}^{I} \gamma_{\perp} \psi_{j}^{J} \bar{\chi} \gamma_{\perp} \Gamma^{I J} \chi \\
& -\frac{1}{4} i \bar{\chi} \chi \epsilon^{i j} \bar{\psi}_{i}^{I} \gamma_{\perp} \psi_{j}^{I}+\frac{1}{8} \sqrt{h}(\bar{\chi} \chi)^{2}-\frac{1}{96} \sqrt{h}\left(\bar{\chi} \gamma^{a} \Gamma^{I J} \chi\right)^{2}
\end{aligned}
$$

At this point, it is still not obvious that this expression can be rendered polynomial. This requires an additional multiplication by the factor $\sqrt{h}$. Using the various relations given already, one arrives at the final result

$$
\begin{aligned}
\sqrt{h} \ddot{H}_{\perp}= & \epsilon^{a b c} \Pi_{a}^{i} \Pi_{b}^{j} F_{i j c}+i h h^{i j} \epsilon_{j k} \Pi_{a}^{k} \bar{\chi} \gamma^{a} D_{i} \chi-\frac{1}{4} \tilde{P}^{A} \tilde{P}^{A}+\frac{1}{4} h h^{i j} P_{i}^{A} P_{j}^{A} \\
& -i \Pi_{a}^{i} \bar{\chi}^{A} \gamma^{a} \psi_{i}^{I} \tilde{P}^{A} \Gamma_{A \dot{A}}^{I}-\frac{1}{2} h h^{i j} \bar{\chi}^{\dot{A}} \psi_{i}^{I} P_{j}^{A} \Gamma_{A \dot{A}}^{I}+2 i \epsilon_{a b c} \Pi^{b i} \Pi^{c j} \bar{\chi}^{\dot{A}} \gamma^{a} \psi_{i}^{I} P_{j}^{A} \Gamma_{A \dot{A}}^{I} \\
& +\frac{1}{4} h h^{i j} \psi_{i}^{I} \gamma^{a} \psi_{j}^{J} \bar{\chi} \gamma_{a} \Gamma^{I J} \chi-\Pi_{a}^{i} \Pi_{b}^{j} \psi_{i}^{I} \gamma^{a} \psi_{j}^{J} \bar{\chi} \gamma^{b} \Gamma^{I J} \chi \\
& -\frac{1}{2} i \epsilon^{a b c} \epsilon_{k l} \Pi_{b}^{k} \Pi_{c}^{l} \epsilon^{i j} \psi_{i}^{l} \gamma^{a} \psi_{j}^{l} \bar{\chi} \chi+\frac{1}{8} h(\bar{\chi} \chi)^{2}-\frac{1}{96} h\left(\bar{\chi} \gamma^{a} \Gamma^{I J} \chi\right)^{2}
\end{aligned}
$$

which is manifestly polynomial (remember that $h h^{i j}=4 \Pi^{a i} \Pi_{a}^{j}$ is polynomial). Again, there is no longer any need for nondegeneracy of the metric at this point.

Finally, the expression for the $E_{8}$-charge $C_{\varepsilon}$ in terms of the new canonical variables reads

$$
\epsilon_{\varepsilon}=\int \mathrm{d}^{2} x \gamma^{\cdot}\left(\tilde{P}^{A} Y^{A}+\left(\epsilon^{i j} \bar{\psi}_{i}^{\prime} \psi_{j}^{J}-\frac{1}{2} i \epsilon^{a b c} \epsilon_{i j} \pi_{a}^{i} \pi_{b}^{j} \bar{\chi} \gamma_{c} \Lambda^{I J} \chi\right)\right) \Upsilon^{\cdot-1}
$$

Again, use of the variable $\tilde{P}^{A}$ leads to a simplification. 
As a useful check on these brackets, one can verify that these constraints generate their respective gauge transformations with time-independent (but, of course, space-dependent) parameters. For the SO(16)-generators, this was already shown in eq. (3.41). It should be kept in mind, however, that these transformations are in general obtained only modulo the constraints. For instance, spatial diffeomorphisms are generated by

$$
\delta_{\xi} \varphi(x)=\left\{\int \mathrm{d}^{2} y \xi^{j} \mathscr{H}_{j}^{\prime}(y), \varphi(x)\right\}
$$

where $\varphi$ stands for any of the fields. As an example, let us consider the variation of the dreibein $V_{i}^{a}$; the above formula yields

$$
\left\{\int \mathrm{d}^{2} y \xi^{j} \mathscr{H}_{j}^{\prime}, V_{i}^{a}\right\}=\partial_{i} \xi^{j} V_{j}^{a}+\xi^{j} \partial_{j} V_{i}^{a}+\epsilon_{i j} \xi^{j} \cdot L^{a}
$$

which coincides with the usual result only on the subspace $L^{a}(x) \approx 0$. Another example is

$$
\left\{\int \mathrm{d}^{2} y \xi^{j} \mathscr{H}_{j}^{\prime}, \tilde{P^{A}}\right\}=\partial_{j}\left(\xi^{j} \tilde{P}^{A}\right)-\frac{1}{2} \Gamma_{A B}^{I J} \xi^{j} P_{j}^{B} \cdot \phi^{I J}
$$

Likewise, the supersymmetry transformations with time-independent parameter $\epsilon^{I}(x)$ can be obtained from

$$
\delta_{\epsilon} \varphi(x)=\left\{\int \mathrm{d}^{2} y \bar{\epsilon}^{I}(y) \mathscr{P}^{I}(y), \varphi(x)\right\}
$$

There is a subtlety, however, when this formula is applied to $\tilde{P}^{A}$, because the formula for $\delta \tilde{P}^{A}$ contains a time derivative and hence cannot be correctly reproduced without use of the equations of motion. I have also verified for some examples that, again modulo the constraints, the equations of motion are generated by the bracket with the hamiltonian constraint.

Given the canonical brackets and the explicit expressions for the constraints derived above, it is now possible to determine the algebra of local and rigid symmetries. It should be noted that, although the constraints are polynomial, the brackets involving $A_{i}^{a}$ and $\chi^{\dot{A}}$ contain inverse factors of $\sqrt{h}$ which seem to vitiate the polynomiality of the structure functions. We can remedy this defect by taking the weighting functions to be appropriate densities as will be exemplified below. The commutator of two Lorentz generators is entirely straightforward to compute; 
we get

$$
\left\{\int \mathrm{d}^{2} x \omega_{1}^{a} L_{a}, \int \mathrm{d}^{2} y \omega_{2}^{b} L_{b}\right\}=\int \mathrm{d}^{2} x\left(-\epsilon^{a b c} \omega_{1 a} \omega_{2 b}\right) L_{c}
$$

For the generators of spatial diffeomorphisms the calculation is equally straightforward, provided one exploits the fact that they generate the respective transformations on the fields. and collects all the extra terms proportional to the constraints if there are any such contributions. In this way one obtains

$$
\begin{aligned}
\left\{\int \mathrm{d}^{2} x \xi_{1}^{i} \ddot{H}_{i}, \int \mathrm{d}^{2} y \xi_{2}^{j} \ddot{F}_{j}^{j}\right\}= & \int \mathrm{d}^{2} x\left(\xi_{2}^{j} \partial_{j} \xi_{1}^{i}-\xi_{1}^{j} \partial_{j} \xi_{2}^{i}\right) \mathscr{H}_{i}^{\prime} \\
& +\frac{1}{2} \xi_{2}^{i} \xi_{1}^{j} P_{i}^{A} P_{j}^{B} \Gamma_{A B}^{I J} \cdot \phi^{I J}+2 \xi_{2}^{i} \xi_{1}^{j} F_{i j a} \cdot L^{a} .
\end{aligned}
$$

This trick may also be used to determine the commutator of $\mathscr{H}_{i}^{\prime}$ with the remaining constraints. The commutator between two $\mathrm{SO}(16)$-transformations was already given in eq. (3.40).

The brackets involving the supersymmetry and the hamiltonian constraints are considerably more involved than in the matter-free case due to the numerous higher-order fermionic terms. For the evaluation of the corresponding brackets, extensive use must be made of the Fierz identities listed in the appendix of ref. [20]. I have completely determined the commutator of two local supersymmetry generators, which is the most important, and performed some partial checks for the hamiltonian constraint (which, to lowest order, give just the expected results). Of course, a full check of the closure of the algebra would also require the complete evaluation of the commutator between two suitably weighted hamiltonian constraints, but I hope that the results presented here are more than sufficient to convince the reader that a complete check would produce neither inconsistencies nor new insights.

The basic formula, which is obtained after a tedious calculation, reads

$$
\begin{aligned}
\left\{\int \mathrm{d}^{2} x \bar{\epsilon}_{1}^{I} \cdot,^{I}, \int \mathrm{d}^{2} y \bar{\epsilon}_{2}^{J} \cdot f^{J}\right\} \\
=-\int \mathrm{d}^{2} x i \bar{\epsilon}_{1}^{I} \gamma^{\mu} \epsilon_{2}^{I} \cdot \not_{\mu}+\frac{1}{8} \int \mathrm{d}^{2} x\left(\bar{\epsilon}_{1}^{I} \epsilon_{2}^{J} \bar{\chi} \Gamma^{I} \Gamma^{K L} \Gamma^{J} \chi-\bar{\epsilon}_{1}^{I} \gamma^{a} \epsilon_{2}^{J} \bar{\chi} \gamma_{a} \Gamma^{I} \Gamma^{K L} \Gamma^{J} \chi\right) \cdot \phi^{K L} \\
\quad+\frac{l}{2} \int \mathrm{d}^{2} x\left(i \bar{\epsilon}_{1}^{\prime} \gamma^{a} \epsilon_{2}^{I} \bar{\chi} \chi-i \bar{\epsilon}_{1}^{I} \epsilon_{2}^{J} \bar{\chi} \gamma^{a} \Gamma^{I J} \chi\right) \cdot L_{a} .
\end{aligned}
$$

Notice that the various other transformations known to arise in the commutator of two local supersymmetries [23] such as another local supersymmetry transforma- 
tion have been compactly assembled into $\mathscr{H}_{\text {, }}$ (see eq. (4.4)), so that, apart from the higher-order fermionic contributions, the commutator has a rather simple structure. The structure functions multiplying $\phi^{\prime J}$ and $L^{a}$ are polynomial. but the first term on the right-hand side is not. Writing $\gamma^{\mu} \ddot{H}_{\mu}=\gamma_{\perp} \mathscr{H}_{-}+\hat{V}_{a}^{i} \gamma^{\alpha} \ddot{H}_{i}$. we see that a factor $h$ is missing. This is not a serious problem as we can multiply the supersymmetry parameters in (4.16) by $\sqrt{h}$ (thereby changing their weights under diffeomorphisms). This procedure produces an extra contribution proportional to $y^{\prime \prime}$ in the above bracket as $\sqrt{h}$ does not commute with $y^{\prime}$. It is not difficult to see that the extra term is also polynomialt.

Next we turn to the brackets containing $\mathscr{C}$. Let us first check the algebra of the $\mathrm{E}_{8}$-charges $\mathscr{E}_{\alpha \beta}$. Using the completeness relations (A.3), one finds

$$
\left\{\mathscr{C}_{\alpha \beta}, \mathscr{C}_{\gamma \delta}\right\}=120\left(\delta_{\alpha \delta} \mathscr{C}_{\beta \gamma}-\delta_{\beta \gamma} \mathscr{C}_{\alpha \delta}\right)+\int \mathrm{d}^{2} x\left(Y^{\cdot} Y^{A} Y^{-1}\right)_{\alpha \beta}\left(Y^{\cdot} Y^{B} Y^{-1}\right)_{\gamma \delta} \Gamma_{A B}^{H S} \cdot \phi^{H} .
$$

The $E_{8}$-algebra in its usual form is recovered after contraction with the $E_{8}$-generators. As expected, the charges generate the $E_{8}$ Lie algebra on the physical subspace where $\phi^{I J}=0$. The brackets between $\mathscr{E}$ and the Lorentz and SO(16)generators simply vanish:

$$
\left\{\phi^{I J}(x), \mathscr{C}\right\}=\left\{L^{a}(x), \mathscr{C}\right\}=0 .
$$

These relations express the invariance of $\mathscr{C}$ under local SO(16) and local Lorentz

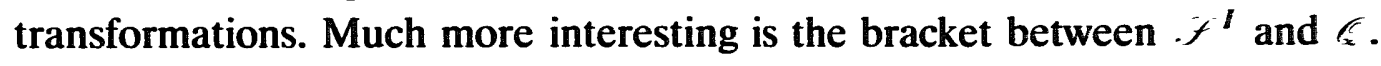

$$
\left\{\mathcal{F}^{I}(x), \mathscr{C}\right\}=-2\left(\mathcal{Y}^{\prime} X^{I J} Y^{-1}\right) \mathscr{F}^{J}(x)+\frac{1}{2}\left(\Gamma^{I} \Gamma^{K L}\right)_{A A}\left(Y^{-} Y^{A} Y^{-1}\right) \chi^{A} \cdot \phi^{K L}(x) .
$$

Observe that the inclusion of the higher-order fermionic terms which were not taken into account in ref. [1], does not alter the result obtained there. The same is true for the commutator with the diffeomorphism constraint:

$$
\left\{\mathscr{H}_{i}^{\prime}(x), \mathscr{C}\right\}=\frac{1}{2} \Gamma_{A B}^{I J} P_{i}^{A}\left(\mathscr{Y} Y^{B} \mathscr{Y}^{-1}\right) \cdot \phi^{I J}(x) .
$$

In evaluating the bracket with the hamiltonian constraint it is advantageous to make use of the fact that the fermionic bilinears in $\mathscr{G}$ generate SO(16)-rotations on the fermions. Since the higher-order fermionic terms in eqs. (4.5), (4.7) and (4.8) are manifestly $\mathrm{SO}(16)$ invariant, they do not contribute to the result. Due to eq. (3.24), the bracket between $A_{i}^{a}$ and $\mathscr{E}$ likewise gives no contribution. The

${ }^{\star}$ Because $\sqrt{h}\left(A_{i}^{a}, \sqrt{h}\right\}=2 \sqrt{h} \epsilon^{a b c} n_{b} V_{c i}$ is polynomial. 
result is

$$
\left\{\sqrt{h} \mathscr{H}_{\perp}(x), \Leftarrow\right\}=\left(\frac{1}{2} \tilde{P}^{A}-i \pi_{a}^{i} \bar{\psi}_{i}^{l} \gamma^{a} \varkappa^{\prime A} \Gamma_{A A}^{I}\right) \Gamma_{A B}^{K L} \mathscr{V} Y^{B} \mathscr{T}^{-1} \cdot \phi^{K L}
$$

The structure functions appearing in eqs. (4.19)-(4.21) depend in a very simple way on the variables $\tilde{P^{A}}, P_{i}^{A}, \eta^{\cdot} Y^{A} y^{-1}$ and $\mathscr{T}^{-1} X^{I J} \mathscr{Y}^{-1}$. Altogether, these results prove that, on the constraint surface $\mathscr{H}_{\mu}(x)=L^{a}(x)=\phi^{I J}(x)=\mathscr{S}^{I}(x)=0$, the charges $\mathscr{C}$ indeed commute with all local symmetries and therefore constitute physical observables in the sense of Dirac.

Commutators similar to eq. (4.19) between local supersymmetry transformations and rigid symmetries have already been evaluated in ref. [25]. The connection with these results is easily established by expanding $\%^{-}$in terms of the scalar fields in the "unitary" gauge $\varphi^{I J}=0$, cf. eq. (3.26). The nonvanishing result of this computation was interpreted as evidence for the existence of a spectrum-generating supergroup, with an infinite tower of new supercharges constructed out of $\mathscr{P}$ and products of the scalar fields. The question here is whether one should regard combinations of field-dependent "structure constants" and constraints such as the right-hand side of (4.19) as symmetry generators in their own right. However, it is obvious that an arbitrary number of such "symmetries" can be generated by taking further commutators. Furthermore, these are all proportional to the constraints and thus carry no additional information.

\section{New canonical variables in $d>4$ dimensions?}

The introduction of new variables in the canonical formulation of gravity was mainly motivated by the fact that in this way the nonlinear hamiltonian constraint could be rendered polynomial [2]. In the preceding sections, a similar simplification was achieved for $N=16$ supergravity. In the light of the results of ref. [19], this is, of course, not a totally unexpected result. Still, it is noteworthy that the simplifications also occur for the higher-order fermionic terms and the highly nonlinear scalar sector, which have not been considered so far in this context. In the scalar sector, it was necessary for this purpose to replace the 128 scalars and their canonically conjugate momenta by the variables $\tilde{P}^{A}, P_{i}^{A}$ and $Q_{i}^{I J}$. For the $\mathrm{E}_{\mathrm{g}}$-charges, one needs in addition the combinations $\gamma^{A} Y^{A} 7^{\cdots-1}$ and $Y^{\top} X^{I J} 7^{\cdots-1}$. I will now argue that it is really the hidden geometrical structure of the higherdimensional theory (here $d=11$ supergravity), which is at the root of the comparative simplicity of the formulas for the constraints and charges. In particular, the fields $\Psi^{A} Y^{A} Y^{-1}, Y^{A} X^{I J} Y^{-1}, P_{i}^{A}$ and $Q_{i}^{I J}$ should be viewed as the remnants of a suitable "vielbein" and connection of the higher-dimensional ancestor of this theory, $d=11$ supergravity. It is here that contact is made with refs. [3,4], where alternative formulations of $d=11$ supergravity with enlarged tangent space sym- 
metries were proposed. An essential ingredient in that construction was the fusion of gravitational and matter degrees of freedom into new tensors covariant under the enlarged symmetry, while the dependence on all eleven coordinates was maintained. This indicates that, although by simple counting arguments, an analogue of Ashtekar's variables for pure gravity in dimensions $d>4$ is unlikely to exist, this may no longer be so if suitable matter degrees of freedom are added. Of course, these extra degrees of freedom would have to conspire with the gravitational degrees of freedom in a rather peculiar way. In this section, some evidence is presented that such a conspiracy does take place if the extra matter degrees of freedom are those required by local supersymmetry. Since the arguments are somewhat preliminary, I will be rather brief. The full canonical treatment of the higher-dimensional theory would require knowledge of the invariant actions, from which the results of refs. $[3,4]$ can be deduced. Up to now, however, only the fermionic part of the $S O(1,3) \times S U(8)$-invariant action has been worked out in ref. [3].

The strongest argument in favor of these ideas is the following. In ref. [3,4] it was demonstrated that the supersymmetry variations of $d=11$ supergravity can be cast into a form, which is covariant with respect to the enlarged tangent space symmetry, with the heip of the "new variables" alluded to above. Significantly, the variations turn out to be polynomial in terms of these variables [3,4]. Because the commutator of two local supersymmetry transformations gives rise to the fermionic equations of motion, from which, in turn, the bosonic equations of motion can be deduced, it follows that all equations are polynomial (this claim has been partially verified by explicit calculation for the $\mathrm{SO}(1,3) \times \mathrm{SU}(8)$-invariant version, $\mathrm{cf}$. sect. 7 of ref. [3]). Therefore, the action, from which these equations follow, must likewise be polynomial. This indicates that the canonical treatment will lead to polynomial constraints.

For the $S O(1,2) \times S O(16)$-invariant version of ref. [4], the reformulation was accomplished by means of "generalized vieibein" $\left(e_{A}^{m}, e_{I J}^{m}\right)$ with upper world indices $m, n, \ldots=3, \ldots, 10$ referring to the "internal" or compactified dimensions, and an $\mathrm{E}_{8}$-valued connection $P_{\mu}^{A}, Q_{\mu}^{I J}$ in eleven dimensions, which are linked to the phase-space variables introduced in sect. 3 (I will ignore the fermions in the remainder of this section). These are the basic objects incorporating the physical bosonic degrees of freedom. They replace those fields of the original version [5] which become scalars in the dimensional reduction to three dimensions, namely the "internal" components $g_{\mu m}, g_{m n}$ and $A_{m n p}, A_{\mu n p}$ of the metric and the three-index tensor, respectively (remember that, inspite of this index split, the new quantities still depend on eleven coordinates). The vielbein $\left(e_{A}^{m}, e_{I J}^{m}\right)$ is related to the inverse metric in the internal space through

$$
e_{A}^{m} e_{A}^{n}+\frac{1}{2} e_{I J}^{m} e_{I J}^{n}=16 \Delta^{-2} g^{m n},
$$

where $\Delta \equiv \operatorname{det} g_{m n}$. It is the analogue of the formula $h h^{i j}=4 \Pi^{a i} \Pi_{a}^{j}$ which played 
an important role in sect. 4. Thus, the metric and its inverse are regarded as derived quantities, whereas the vielbein is considered fundamental. It is, of course, essential here that neither the metric nor its inverse ever appear in the formulas of refs. $[3,4]$. The similarity with the results of ref. [2] is rather striking.

The generalized vielbein is subject to a number of constraints. The following ones, which are algebraic, were not given in ref. [4]

$$
\Gamma_{A B}^{\prime J} e_{A A}^{m} e_{B}^{n}+4 e_{\mathcal{K} \mid l}^{m} e_{J] K}^{n}=0, \quad \Gamma_{A B}^{J J}\left(e_{B}^{m} e_{I J}^{n}-e_{B}^{n} e_{I J}^{m}\right)=0 .
$$

They are easy to prove in the special gauge adopted in ref. [4] (see eq. (20)), and hence generally valid by virtue of SO(16)-covariance. The vielbein components satisfy a generalization of the vielbein postulate of general relativity, which takes the form

$$
D_{i} e_{A}^{m}+\frac{1}{4} \Gamma_{A B}^{J J} P_{i}^{B} e_{I J}^{m}=0, \quad D_{i} e_{I J}^{m}-\frac{1}{2} \Gamma_{A B}^{J J} P_{i}^{A} e_{B}^{m}=0 .
$$

Although the index $i$ has been assumed to refer to two spatial dimensions until now, the above relations are, in fact, valid in eleven dimensions (I have taken the liberty to ignore the terms with $\partial_{m} B_{\mu}^{n}$ in eq. (25) of ref. [4], since these vanish in the dimensional reduction to be considered below). Obviously, these relations are analogous to the conditions expressing the covariant constancy of the soldering form in ref. [2].

To establish the connection with the results of the foregoing sections, we must now perform the dimensional reduction, dropping the dependence on the "internal" coordinates (this is sometimes referred to as torus reduction, being the simplest kind of Kaluza-Klein compactification). This is straightforward for $\boldsymbol{P}_{i}^{\boldsymbol{A}}$ and $Q_{i}^{\prime J}$ which can be directiy identified with the corresponding fields here. To express the vielbein components $e_{A}^{m}$ and $e_{I J}^{m}$ through $\gamma^{\prime} Y^{A} 7^{-1}$ and $Y^{\prime} X^{I J} Y^{-1}$ is. however, slightly more tricky. To this end we pick eight generators $t^{m}$ of the Cartan subalgebra of $E_{\$}$ which are orthonormal with respect to the Cartan-Killing metric. Then all the above relations are satisfied with the identification

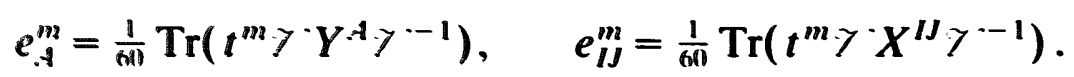

Let us first check the generalized vielbein postulate; it is a consequence of

$$
\begin{aligned}
& D_{i}\left(y^{A} Y^{-1}\right)=-\frac{1}{4} P_{i}^{B} \Gamma_{A B}^{I J}\left(Y^{\prime} X^{I J} y^{-1}\right), \\
& D_{i}\left(Y^{\prime} X^{I J} Y^{-1}\right)=+\frac{1}{2} \Gamma_{A B}^{I J} P_{i}^{A}\left(y^{B} Y^{B-1}\right) .
\end{aligned}
$$

To prove the algebraic relations (5.2), one substitutes (5.4) and makes use of the completeness relations (A.3); this leads for instance to

$$
\Gamma_{A B}^{I J} e_{A}^{m} e_{B}^{n}+4 e_{K \mid I}^{m} e_{J \mid K}^{n}=-120 \operatorname{Tr}\left(\left[t^{m}, t^{n}\right] \Upsilon^{\prime} X^{I J} Y^{-1}\right),
$$

It is here that we must assume the generators $t^{m}$ to span the Cartan subalgebra of $E_{s}$, since otherwise the right-hand side of (5.6) would fail to vanish. Incidentally, 
these formulas furnish an explicit expression for the metric ansatz in terms of the 128 scalars which follows simply by substitution of (5.4) into (5.1). The formula (5.4) makes the role of the vielbein quite explicit: just as $\mathscr{V}$ converts (local) SO(16) indices into (rigid) $\mathrm{E}_{8}$ indices, the fields $\left(e_{A}^{m}, e_{I j}^{m}\right)$ convert $\mathrm{SO}(16)$ tangent space indices into (internal) world indices; for this reason, it would also be appropriate to call them "soldering forms" with respect to the enlarged tangent space group.

To go beyond these remarks and actually demonstrate the usefulness of the "new variables" in a canonical treatment of $d=11$ supergravity or other higher dimensional theories will require a lot more work, but $I$ hope that the arguments presented above are sufficient to convince the reader that it would be a worthwhile endeavor.

\section{Discussion}

One of the important open problems concerns the quantization of the theory. Treating all fields as operators, the phase-space constraints (3.14) must now be replaced by operator constraints on the physical states $\Psi$. By analogy with ordinary supersymmetric quantum field theories, we expect that the high degree of symmetry of extended supergravities will alleviate quantum ordering problems. For instance, a glance at (4.1) and the canonical brackets (now commutators or anticommutators) shows that there are no such ordering problems for $\mathscr{f}^{l}$. As all other operators appear in the anticommutator of two supersymmetry generators, we can simply define the ordering of the other operators through the way in which they appear in eq. (4.16) - assuming the absence of anomalies on the right-hand side of eq. (4.16). The equations with dynamical content are the Wheeler-DeWitt equation $\mathscr{H}_{\perp}(x) \Psi=0$ and its "square-root"

$$
\mathscr{P}^{\prime}(x) \Psi=0 \text {. }
$$

Any solution of this constraint will automatically satisfy all of the other constraints by (4.16). At least formally, one can generate new solutions from one particular solution through the action of $\mathscr{C}$, because $\mathscr{C}$ and $\mathscr{S}^{l}$ commute on-shell by eq. (4.19). Of course, we must here assume that the order of the terms on the right-hand side of (4.19) can be maintained in the quantum theory; otherwise there will be extra anomalous contributions which invalidate this argument. With this assumption, the solutions of (6.1) clearly belong to multiplets of the rigid symmetry group. It is interesting to speculate about the cosmological interpretation of the associated conserved quantum numbers.

In principle, it should be possible to represent the condition in eq. (6.1) as a functional differential equation, which is polynomial in the fundamental operators. However, in trying to replace the canonical brackets of sect. 3 by appropriate operator commutation or anticommutation relations, one encounters some technical obstacles, which I have not been able to overcome so far. These complications are entirely due to the matter sector. For instance, it is obvious that, in the gauge $n^{a}=(1,0,0)$, eq. (3.17) can be easily converted into an operator relation by 
redefining the spinors according to $\chi^{\dot{A}} \rightarrow h^{1 / 4} \chi^{\dot{A}}$. If, on the other hand, $n^{a}$ does depend nontrivially on the dreibein $V_{i}^{a}$, it is not clear how to proceed. Further problems are causeci by the $\bar{x} x$ term on the right-hand side of eq. (3.22), which apparently cannot be removed by a redefinition of $A_{i}^{a}$.

In order to make progress at this point it may be necessary to study the reduction of (6.1) to two space-time dimensions, where the hidden rigid symmetries become infinite dimensional $[12,26,27]$. The associated conserved current, which has been found in ref. [1], now gives rise to infinitely many charges and thus to infinitely many observables. We can therefore apply an infinite set of operators to an arbitrarily chosen solution $\Psi$ of eq. (6.1) in order to generate new solutions. This suggests that quantum-extended supergravities are completely integrable in two dimensions [1]. Note that this notion of integrability is very different from the one prevalent in investigations of stationary axisymmetric solutions of Einstein's equations, where one studies the classical equations of motion [26]. However, in the presence of Grassmann-valued fermions, it does not make much sense to talk about classical solutions (at least in the author's opinion). It is much more meaningful to address this question in the quantum-mechanical context outlined above.

It is also interesting to note that the construction of the conserved charges for the $d=2$ theory relies on the existence of a linear system (or Lax pair) for $d=2$ supergravity [27]. This linear system furnishes a curvature-free connection involving both gravity and matter fields which has no analogue for $d>2$ (and would be necessary there for the survival of the Wilson loop as an observable). It thus appears that the topological observables of pure gravity and the more conventional observables considered in this paper are actually related in the two-dimensional reduction. It has also been conjectured that the reduction to one dimension will reveal the existence of hyperbolic symmetries, and in particular an $\mathrm{E}_{10}$-symmetry for maximally extended supergravity [12]. If this is true, the classification of observables in quantum (super)gravity may be linked to the construction of representations of hyperbolic algebras.

A considerable part of this work was done during a stay at KEK. I would like to thank the KEK Theory Group and especially K. Higashijima for their hospitality.

\section{Appendix A}

\section{$\mathrm{E}_{8}$ CONVENTIONS}

The $\mathrm{E}_{8}$ Lie algebra has 128 symmetric generators $Y^{A}$ and antisymmetric generators $X^{I J}$ in its fundamental 248 dimensional representation. We collectively

\# Because of the Majorana condition on $x^{\dot{A}}$ the manifest SO(16)-covariance must, however, be sacrificed. 
denote the indices $(A, I J)$ by Greek letters $\alpha, \beta, \ldots$. The generators in the fundamental 248 dimensional representation are normalized according to

$$
\operatorname{Tr}\left(Y^{A} Y^{B}\right)=60 \delta^{A B}, \quad \operatorname{Tr}\left(X^{I J} X^{K L}\right)=-120 \delta_{K L}^{I J} .
$$

The generators $X^{I J}$ are therefore compact, while the generators $Y^{A}$ are noncompact. The $\mathrm{E}_{8}$ commutation relations are

$$
\begin{aligned}
{\left[X^{I J}, X^{K L}\right] } & =\delta^{J K} X^{I L}+\delta^{I L} X^{J K}-\delta^{I K} X^{J L}-\delta^{J L} X^{I K} \\
{\left[Y^{A}, Y^{B}\right] } & =\frac{1}{4} \Gamma_{A B}^{I J} X^{I J}, \quad\left[X^{I J}, Y^{A}\right]=-\frac{1}{2} \Gamma_{A B}^{I J} Y^{B} .
\end{aligned}
$$

We have the following completeness relations

$$
\begin{gathered}
\frac{1}{60} Y_{\alpha \beta}^{A} Y_{\gamma \delta}^{A}+\frac{1}{120} X_{\alpha \beta}^{I J} X_{\gamma \delta}^{I J}=\delta_{\alpha \gamma} \delta_{\beta \delta} \\
\frac{1}{60}\left(\Gamma_{A B}^{I J} Y_{\alpha \beta}^{A} Y_{\gamma \delta}^{B}-4 X_{\alpha \beta}^{K[I} X_{\gamma \delta}^{J] K}\right)=2\left(\delta_{\alpha \gamma} X_{\beta \delta}^{I J}+\delta_{\beta \delta} X_{\alpha \gamma}^{I J}\right) \\
\frac{1}{60} \Gamma_{A B}^{I J}\left(Y_{\alpha \beta}^{B} X_{\gamma \delta}^{I J}+X_{\alpha \beta}^{I J} Y_{\gamma \delta}^{B}\right)=-4\left(\delta_{\alpha \gamma} Y_{\beta \delta}^{A}-\delta_{\beta \delta} Y_{\alpha \gamma}^{A}\right)
\end{gathered}
$$

These relations are, of course, only valid when traced with elements of the $\mathrm{E}_{8}$ Lie algebra and can be simply verified by contraction with the generators $Y^{A}$ and $X^{I J}$.

\section{References}

[1] H. Nicolai, Phys. Lett. B235 (1990) 295

[2] A. Ashtekar, Phys. Rev. Lett. 57 (1986) 2244;

A. Ashtekar, New perspectives in canonical gravity (Bibliopolis, 1988)

[3] B. de Wit and H. Nicolai, Phys. Lett. B155 (1985) 47; Nucl. Phys. B274 (1986) 363

[4] H. Nicolai, Phys. Lett. B187 (1987) 316

[5] E. Cremmer, B. Julia and J. Scherk, Phys. Lett. B76 (1978) 409

[6] S. Deser, R. Jackiw and G. 't Hooft, Ann. Phys. (NY) 152 (1984) 220;

S. Deser and R. Jackiw, Ann. Phys. (NY) 153 (1984) 405;

J. Abbott, S. Giddings and K. Kuchar, Gen. Rel. Grav. 16 (1984) 751

[7] E. Witten, Nucl. Phys. B311 (1988) 46;

S.P. Martin, Nucl. Phys. B327 (1989) 178

[8] A. Ashtekar, V. Husain, C. Rovelli, J. Samuel and L. Smolin, Class. Quantum Grav. 6 (1989) L185

[9] I. Bengtsson, Phys. Lett. B220 (1989) 51

[10] E. Cremmer, S. Ferrara and J. Scherk, Phys. Lett. B74 (1978) 61;

E. Cremmer and B. Julia, Nucl. Phys. B159 (1979) 141

[11] P. Breitenlohner, D. Maison and G.W. Gibbons, Commun. Math. Phys. 120 (1988) 295 and references therein

[12] B. Julia, in Superspace and supergravity, ed. S.W. Hawking and M. Rocek (Cambridge Univ. Press. Cambridge, 1980); preprint LPTENS 82/22 (1982); in Unified theories in more than four dimensions, ed. V. DeSabbata and E. Schmutzer (World Scientific. Singapore, 1983)

[13] P.A.M. Dirac, Lectures on quantum mechanics (Academic Press, London. 1967)

[14] J.A. Wheeler, in Relativity, groups and topology, ed. C. DeWitt and B. DeWitt (Gordon and Breach, New York. 1964);

B. DeWitt, Phys. Rev. 160 (1967) 113; 162 (1967) 1195 
IISI C. Misker. K. Thorne and J.A. Wheeler, Gravitation (Freeman. San Francisco, 1973)

[16] C. Wham, m General relatixity and gravitation (Cambridge Univ. Press, Cambridge, 1987)

[1.] T. Jitwohson and L. Snolin. Nucl. Phys. B299 (1988) 295:

C. Rovelli and L. Smolin. Nucl. Phys. B331 (1990) 80;

M.P. Blencowe. Nucl. Phis. B.341 (1990) 213

[18] T. Jacobson. Class. Quantum Grav. 5 (1988) 923

[19] A. Ashtekar. J.D. Romano and R.S. Tate, Phys. Rev. D40 (1989) 2572:

T. Jacobson, Class. Quantum Grav. 5 (1988) L143

[20] N. Marcus and J.H. Schwarz, Nucl. Phys. B228 (1983) 145

[21] S. Deser. J.H. Kay and K.S. Stelle. Phys. Rev. D16 (1977) 2448;

E.S. Fradkin and M.A. Vasiliev, Phys. Lett. B72 (1977) 70:

M. Pilati. Nucl. Phys. B132 (1978) 138

[22] A. Achucarro and P.K. Townsend. Phys. Lett. B229 (1989) 383

[23] P. van Nieuwenhuizen. Phys. Rep. 68 (1981) 189

[24] J.E. Nelson and C. Teitelhoim. Phys. Lett. B69 (1977) 81

[25] J. Ellis, M.K. Gaillard and B. Zumino, Phys. Lett. B94 (1981) 221:

N. Marcus, A. Sagnotti and J.H. Schwarz. Nucl. Phys. B243 (1984) 335

[26] R. Geroch, J. Math. Phys. 12 (1971) 918: 13 (1972) 394:

IV. Kinnersley. J. Math. Phys. 14 (1973) 651:

V.A. Belinski and V.E. Sakharox. Zh. Eksp. Theor. Fiz. 75 (1978) 1955: 77 (1979) 3;

D. Maison. Gen. Rel. Grav. 10 (1979) 717:

F. Ernst and I. Hauser. J. Math. Phys. 21 (1980) 1126; 22 (1981) 1051:

P. Breitenlohner and D. Maison. Ann. Inst. Poincare 46 (1987) 215

[27] H. Nicolai. Phys. Lett. B194 (1987) 402:

H. Nicolai and N.P. Warner. Commun. Math. Phys. 125 (1989) 384 